Letters to the Editor

\section{Enterococcal Bacteremia in Cancer Patients}

\section{To the Editor:}

Enterococci are emerging pathogens in cancer patients with neutropenic fever. ${ }^{1}$ The increasing incidence of enterococcal infection may, in part, reflect prophylaxis with quinolones and empirical therapy with third-generation cephalosporins, as both antimicrobials have weak or no activity against enterococci. ${ }^{2,3}$ As part of the national survey of enterococcal bacteremia in Slovakia (January 1, 1997-January 1, 2000), we collected 132 cases of enterococcal bacteremia, including 59 patients (45\%) with cancer as the underlying disease; 55 were due to Enterococcus faecalis and 4 to $E$ faecium. All $E$ faecium isolates were van-resistant exhibiting the van-B phenotype (minimum inhibitory concentration, $16-32$ $\mu \mathrm{g} / \mathrm{mL}$ ). These patients had received quinolone prophylaxis and were pretreated with third-generation cephalosporins, and 2 of 4 had received imipenem or vancomycin (empirically).

We compared characteristics of the 59 enterococcal bacteremia patients with cancer and the 73 patients without cancer, and found that enterococci from the cancer patients were significantly more likely to be resistant to ampicillin, vancomycin, and teicoplanin but were less likely to be resistant to co-trimoxazole or tetracycline (Table). We also found, not unexpectedly, that the cancer patients were significantly more likely to have had prior antibiotic therapy, neutropenia, and chemotherapy, all factors that may contribute to the resistance patterns seen. Mortality was comparable in both groups (33\%-34\%).

Our findings suggest that, among cancer patients at high risk of enterococcal bacteremia (eg, those with prolonged neutropenia pretreated with quinolones, cephalosporins, and carbapenems), resistance to multiple antimicrobials may appear. Therefore, their initial coverage should consist of a combination of anti-enterococcal antimicrobial agents such as piperacillin and gentamicin. If $v a n$ resistance appears, quinupristin-dalfopristin or chloramphenicol should be added.

TABLE

Enterococcal Bacteremia in Cancer Versus Non-Cancer Patients

\begin{tabular}{|c|c|c|c|c|c|c|}
\hline \multirow[b]{2}{*}{ Characteristics } & \multirow[b]{2}{*}{ Total } & \multicolumn{2}{|c|}{ C } & \multicolumn{2}{|l|}{ D } & \multirow{2}{*}{$\frac{\text { C vs D }}{P}$} \\
\hline & & Cancer & $\%$ & Non-Cancer & $\%$ & \\
\hline No. of patients & 132 & 59 & & 73 & & \\
\hline \multicolumn{7}{|l|}{ Microbiology } \\
\hline Enterococcus faecalis & 117 & 54 & 91.5 & 63 & 86.3 & NS \\
\hline Enterococcus faecium & 12 & 5 & 8.5 & 7 & 9.6 & NS \\
\hline Enterococcus gallinarum & 3 & 0 & 0.0 & 3 & 4.1 & NS \\
\hline AMP-resistance & 34 & 24 & 40.7 & 10 & 13.7 & .0009 \\
\hline GEN-resistance & 29 & 12 & 20.3 & 17 & 23.3 & NS \\
\hline VAN-resistance & 9 & 8 & 13.6 & 1 & 1.4 & .0107 \\
\hline TET-resistance & 40 & 6 & 10.2 & 34 & 46.6 & $<.0001$ \\
\hline CMP-resistance & 5 & 4 & 6.8 & 1 & 1.4 & NS \\
\hline COT-resistance & 44 & 10 & 16.9 & 34 & 46.6 & .0007 \\
\hline TEI-resistance & 7 & 7 & 11.9 & 0 & 0.0 & .0029 \\
\hline \multicolumn{7}{|l|}{ Clinical characteristics } \\
\hline 1 positive $\mathrm{BC}$ & 45 & 19 & 32.2 & 26 & 35.6 & NS \\
\hline 2 positive $\mathrm{BC}$ & 58 & 26 & 44.1 & 32 & 43.8 & NS \\
\hline$\geqslant 3$ positive $\mathrm{BC}$ & 29 & 13 & 22.0 & 16 & 21.9 & NS \\
\hline Other body sites & 31 & 11 & 18.6 & 20 & 27.4 & NS \\
\hline Vascular catheter infection & 16 & 6 & 10.2 & 10 & 13.7 & NS \\
\hline Wound infection & 3 & 0 & 0.0 & 3 & 4.1 & NS \\
\hline Urinary tract infection & 13 & 6 & 10.2 & 13 & 17.8 & NS \\
\hline \multicolumn{7}{|l|}{ Risk factors } \\
\hline Vascular catheter & 104 & 42 & 71.2 & 62 & 84.9 & NS \\
\hline Dialysis & 8 & 0 & 0.0 & 8 & 11.0 & .0085 \\
\hline Low birth weight neonate & 13 & 0 & 0.0 & 13 & 17.8 & .0018 \\
\hline Ventilatory support & 35 & 6 & 10.2 & 29 & 39.7 & .0003 \\
\hline Surgery & 57 & 16 & 27.1 & 41 & 56.2 & .0015 \\
\hline Corticosteroid therapy & 12 & 7 & 11.9 & 5 & 6.8 & NS \\
\hline Neutropenia & 38 & 38 & 64.4 & 0 & 0.0 & $<.0001$ \\
\hline Antineoplastic chemotherapy & 48 & 48 & 81.4 & 0 & 0.0 & $<.0001$ \\
\hline Urinary tract surgery & 3 & 0 & 0.0 & 3 & 4.1 & NS \\
\hline GI surgery & 22 & 6 & 10.2 & 16 & 21.9 & NS \\
\hline Burns or decubiti & 8 & 1 & 1.7 & 7 & 9.6 & NS \\
\hline Diabetes & 12 & 4 & 6.8 & 8 & 11.0 & NS \\
\hline Prior antibiotic therapy & 89 & 59 & 100.0 & 30 & 41.1 & $<.0001$ \\
\hline Prior prophylaxis & 33 & 16 & 27.1 & 17 & 23.3 & NS \\
\hline \multicolumn{7}{|l|}{ Outcomes } \\
\hline Endocarditis & 23 & 4 & 6.8 & 19 & 26.0 & .0076 \\
\hline Liver abscess & 4 & 0 & 0.0 & 4 & 5.5 & NS \\
\hline Lung abscess & 10 & 3 & 5.1 & 7 & 9.6 & NS \\
\hline Cured & 88 & 39 & 66.1 & 49 & 67.1 & NS \\
\hline Died & 44 & 20 & 33.9 & 24 & 32.9 & NS \\
\hline
\end{tabular}

Abbreviations: AMP, ampicillin; BC, blood culture; CMP, chloramphenicol; COT, co-trimoxazole; GEN, gentamicin; GI, gastrointestinal; NS, not significant; TEI, teicoplanin; TET, tetracycline; VAN, vancomycin.

\section{REFERENCES}

1. Goosen SH. Clinical epidemiology of glycopeptide-resistant enterococci in Europe. In: Brun-Buisson C, Eliopoulos G, Leclerq R, eds. Bacterial Resistance to Glycopeptides. Flammarion, Paris, France: Medicine Sciences; 1998.

2. Edmond MB, Ober JF, Weinbaum DL, Pfaller MA, Hwang TG, Sanford MD. Vancomycin resistant Enterococcus faecium bacteremia: risk factors for infection. Clin Infect Dis 1995;20:1126-1133.

3. Garbutt JM, Veertrapragda M, Littenberg B, Mundy L. Association between resistance to vancomycin and death in cases of Enterococcus faecium bacteremia. Clin Infect Dis 2000;30:466-472.

E. Bilíková, MSc

J. Hanzen, MD

I. Svetlansky, $\mathrm{PhD}$

M. Lisková, MD, PhD

A. Roidová, MD

V. Krcméry, MD, FACP

University of Trnava

Bratislava, Slovakia 